\title{
Planning of Sustainable Industrial Zones and Means of Achieving them in Iraq
}

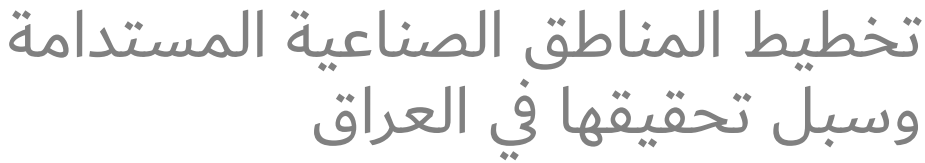

\section{Suaad Jaber Laffta and Areaj Khairy Othman Al-rawi}

\section{إعاد جابر لفتة و اريج خيري عثمان الراوي}

Center of Urban Regional \& Regional planning for postgraduate studies, University of Baghdad مركز التخطيط الحضري والاقليمي للدراسات العليا، جامعة بغداد

Corresponding Author: Suaad Jaber Laffta J_suaad@yahoo.com

Received: 28 December 2017 Accepted: 2 February 2018 Published: 1 May 2018

Publishing services provided by Knowledge

(c) Suaad Jaber Laffta and Areaj Khairy Othman Al-rawi. This article is distributed under the terms of the Creative Commons Attribution License, which permits unrestricted use and redistribution provided that the original author and source are credited.

Selection and Peer-review under the responsibility of the Urban Planning Iraq Conference Committee.

\section{Abstract}

The development of industrial activity in cities and regions is one of the most distinctive features of post-modern urban planning. The industry in all its branches is considered one of the most important economic activities in cities, which is the main engine of economic life and the driving force of development at all levels. This development has been accompanied by the negative effects of this economic activity on ecosystems and on human life in its various aspects, namely, environmental pollution in various ways, depletion of renewable and depleted natural resources, and the serious consequences of climate change, environmental degradation and Collapse. Hence the need to find solutions that allow the continuation of industries because of the importance of human life and to overcome the problems resulting from them and reduce them as much as possible The emergence of the concept of sustainability as a solution to this problem. This paper discusses the theme of sustainable industry, sustainable industrial zone planning, global experiences and identification of deliberate strategies for sustainability, As well as trends in Iraq towards the establishment of green industrial cities, And their analysis and knowledge of their shortcomings in comparison to international experiences. And recommend some policies to achieve sustainability.

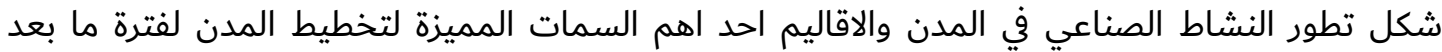

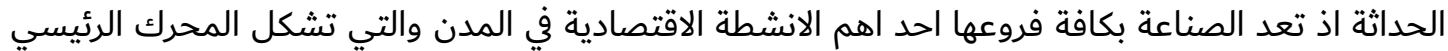

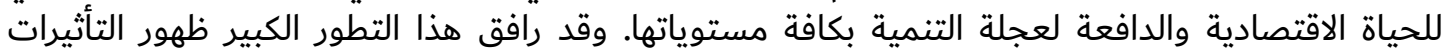

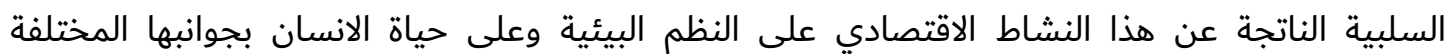

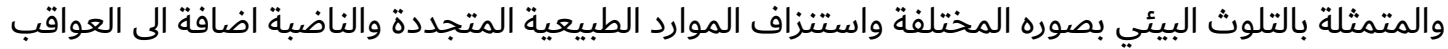

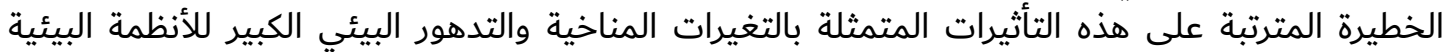

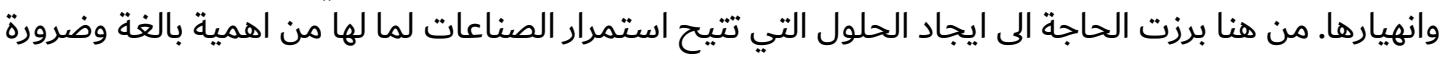

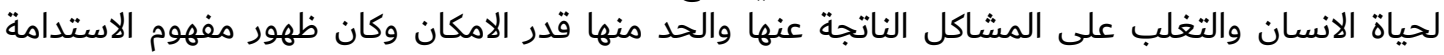

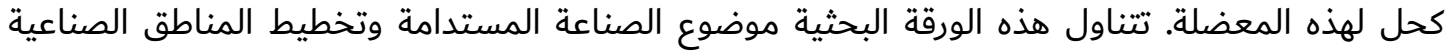

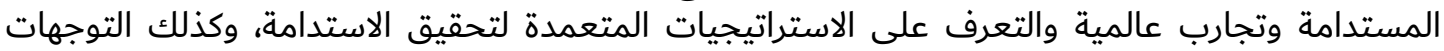

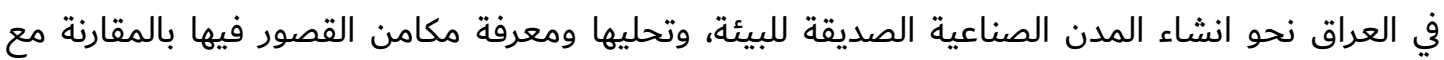
التجارب العالمية. والتوصية ببعض السياسات للتوصل الى الاستدامة. 
Keywords: Sustainable industry; Sustainable industrial zones.

الكامات المفتاحية: الصناعة المستدامة; المناطق الصناعية المستدامة.

يعد قطاع الصناعة الركيزة الاساسية للتنمية التي يعتمد عليها تقدم وتطور الدول، لذا فان من الضروري

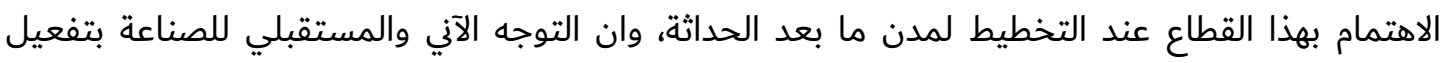

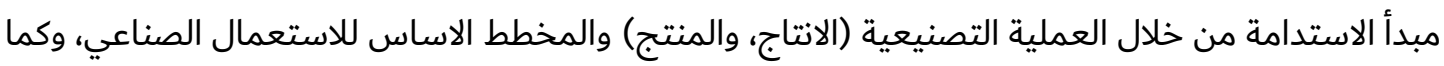
سيتم تناوله في الفقرات اللاحقة.

\section{r. الصناعة المستدامة}

يمكن تعريف الصناعة المستدامة عندما تنتج السلع والخدمات بطريقة تفي بها احتياجات وتطلعات الحاضر دون المساس بقدرة الأجيال المقبلة لتلبية احتياجاتهم الخاصة. ويمكن توضيح ميزات الصناعة المستدامة من خلال:

• قابلة الاستمرار اقتصاديا (يستخدم رأس المال الطبيعي والمالي والبشري لخلق القيمة والثروة والأرباح). • التوافق البيئي (استخدام اساليب الانتاج الأنظف، ومنع التلوث، وتقليل استنزاف الموارد الطبيعية، فضلا عن الحفاظ على التنوع البيولوجي والحياة البئية البرية للموائل). • المسؤولية الاجتماعية (من خلال ادارة مختلف الآثار المترتبة من عملية الإنتاج من خلال المبادرات مثل الرعاية المسؤولة التشاركية). مقومات الصناعة المستدامة ويشمل عملية الإنتاج والمنتج وكالاتي:

\section{r-1. عمليات ومراحل الإنتاج التي تتضمن:}

• توفير التكاليف وتكون أكثر ربحية لأنها أقل إهدار للمواد والطاقة (مما يؤدي إلى انبعاثات أقل من

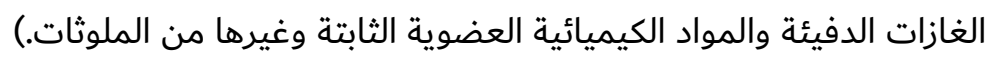
• تمكين استخدام الموارد المتجددة بصورة أكبر وأكثر كفاءة (الطاقة والمواد الكيميائية و المواد)،

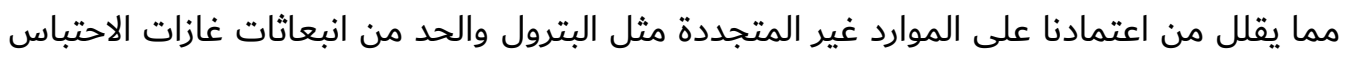
الحراري المرتبطة بها.

r-r. المنتجات التي تمتاز ب: • • أفضل أداء، وأكثر دواما. 
• أقل سمية، وأكثر سهولة لإعادة التدوير وأكثر قابلية للتحلل من نظرائها المنتجة بالطرق التقليدية. • مستمدة قدر المستطاع من الموارد المتجددة وتساهم في الحد الأدنى من انبعاثات غازات الاحتباس

الحراري [1].

\section{"ا. تخطيط الموقع الرئيسي للمناطق الصناعية المستدامة}

ان تخطيط الموقع يتطلب تكامل العوامل البيئية والاجتماعية والاقتصادية. وان التنفيذ الناجح لاستدامة المنطقة الصناعية يتطلب عملية تخطيط شاملة ومفصلة التي تراعية التاعي البئية البئة، والقضايا الاجتماعية والاقتصادية منذ البدء بالعملية التخطيطية.

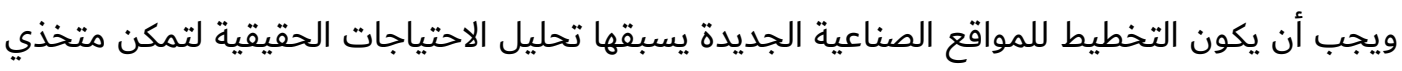

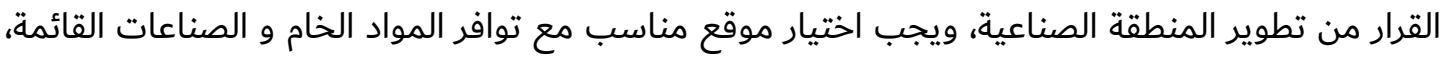

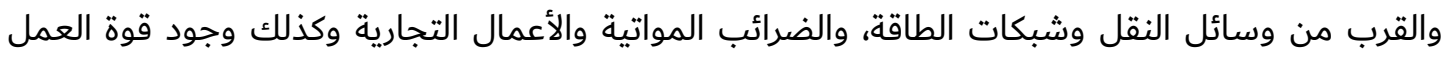

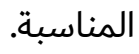

الأد بناء أو إعادة تأهيل منطقة صناعية تحتاج إلى تقييم الآثار البيئية السلبية الناجمة.. ويحتاج تقييم

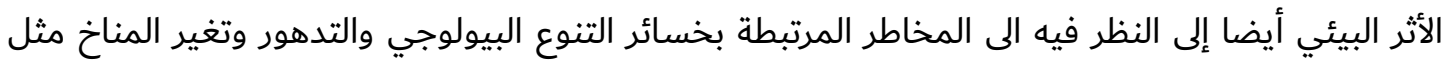

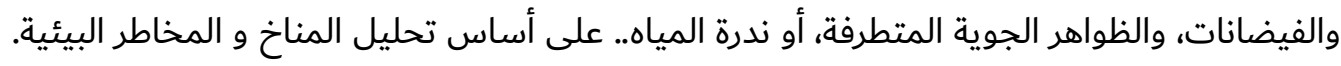

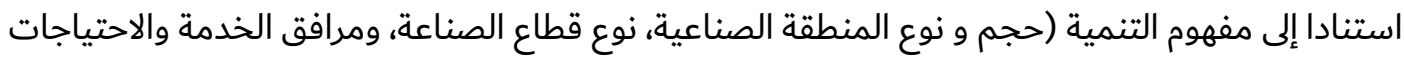
اللازمة لحماية البيئة، والمرافق الاجتماعية المطلوبة، وما إلى ذلك) التخطيط الرئيسي يحتاج ان يتضمناعناف

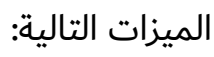

$$
\begin{aligned}
& \text { تكامل المنطقة الصناعية مع البنية التحتية للمحيط. } \\
& \text { - الاستخدام الكفوء للأراضي - } \\
& \text { - توفير البنية التحتية والنقل } \\
& \text { - توليد الطاقة وتوزيعها } \\
& \text { - إدارة المياه والصرف الصحي - } \\
& \text { - } \\
& \text { - إدارة مخاطر الكوارث } \\
& \text { - البنية التحتية الاجتماعية }
\end{aligned}
$$

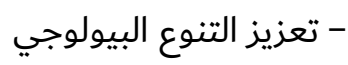

وينظر في وضع المخطط العام للموقع جميع الحقوق المتعلقة بالأراضي والقوانين البيئية فضلا عن

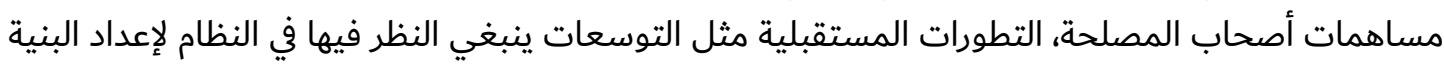
التحتية وفقا لذلك والحد من التكاليف في المستقبل [؟]. 


\section{ع. كفاءة استعمالات الأراضي للمنطقة الصناعية}

يعتمد مفهوم الكفاءة على العلاقة بين المتغيرات الآتية (المصنع / كفاءة استعمالات الأراضي /توفر المساحات الخضراء والمفتوحة/ تكامل المساحات.

خلال التخطيط الرئيسي للمناطق الصناعية الجديدة يجب وضع مفاهيم للاستعمال الفعال للأراضي

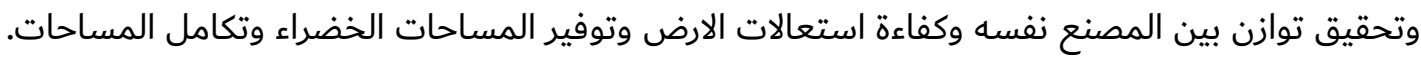
عند قيام صناعة جديدة لابد من تفعيل مفهوم استعمالات الأراضي فان العلاقة بين المباني والمساحات الخضراء المفتوحة هو المهم. ولا بد من ضمان استعمال الأرض وكفاءة الفضاءات المتجاورة للتحسين من المناخ، وحماية التنوع البيولوجي واستجمام الناس العاملين في الموقع ولان وهذا يحتاج إلى بعض القواعد التنظيمية في التخطيط والبناء مثل أقصى ارتفاعات المباني، تنظيم استعمالات الأراضي والعلاقات مع مساحة السطوح.

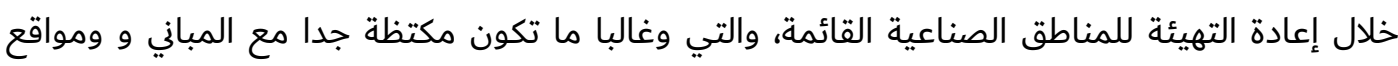

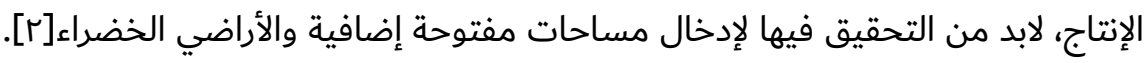

\section{0. المناطق او المجمعات الصناعية البيئية}

يعرف المجمع البيئي الصناعي باعتباره نظاما صناعيا يحافظ على الموارد الطبيعية والاقتصادية. ويقلل من الإنا

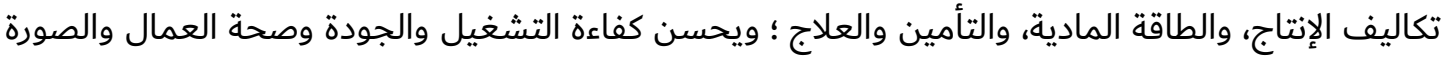

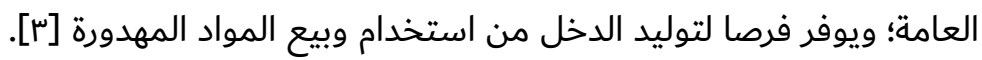

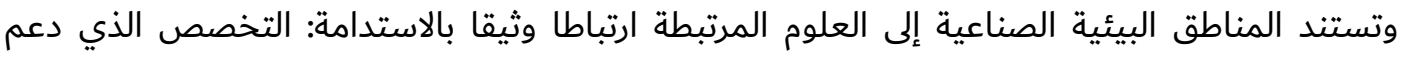

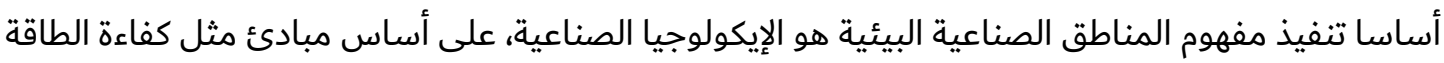
(التي تتبنى الاستخدام الفعال للموارد الطبيعية من أجل تلبية حاجات الإنسات الإنسان) حلقات المواد الاولية المغلقة (التي تسعى لتحقيق التوازن بين المدخلات والمخرجات عن طريق تقليل

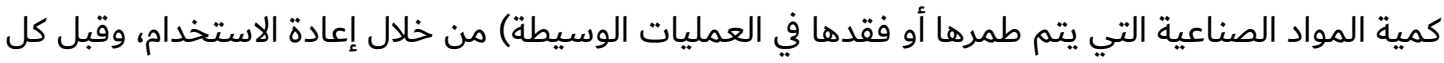

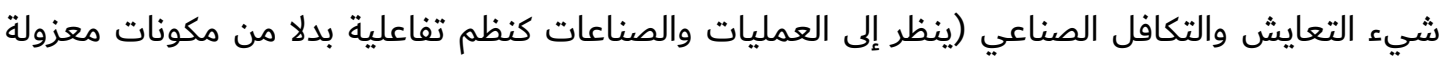

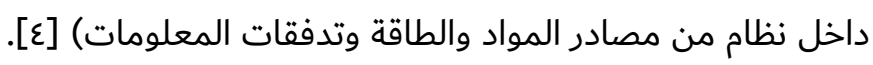

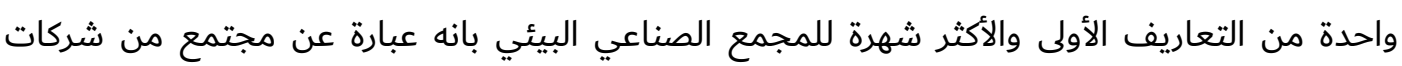

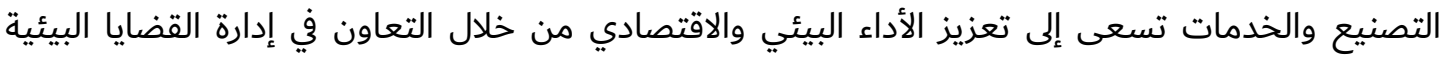

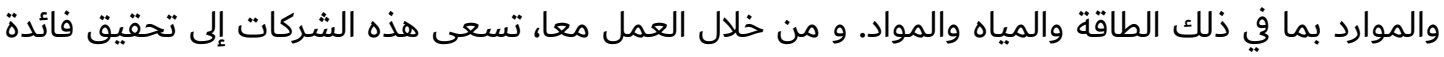

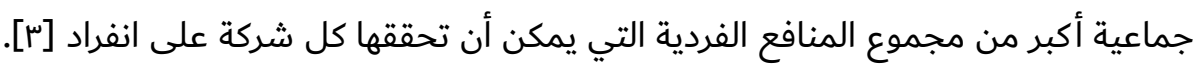

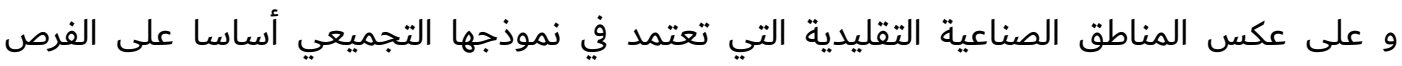
الاقتصادية أو تقرر ببساطة توطين نفسها في مناطق يسهل الوصول إليها، على مقربة من البنى التحتية التئية

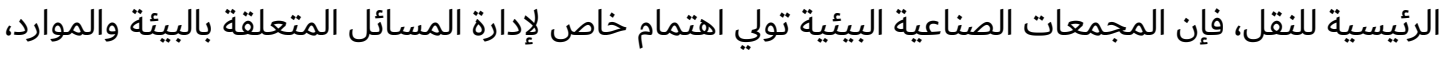

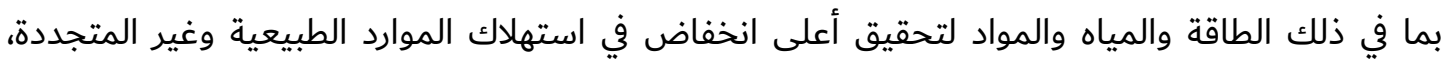
والانبعاثات والنفايات [0]. 
وفي إطار التخطيط، يمكن أن يكون الاختلاف الرئيسي بين المنطقة الصناعية البيئية والمنطقة الصناعية

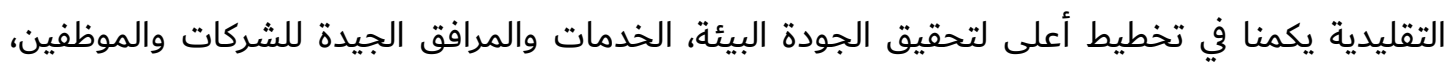

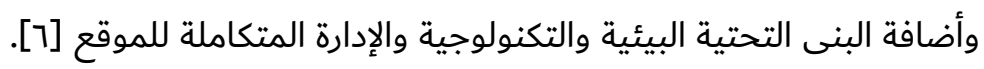

\section{7. الاستراتيجيات الرئيسية لتطوير الحدائق الصناعية الايكولوجية}

هنالك العديد من الاسترتيجيات الممكن اعتمادها لتطوير الحدائق الصناعية [V]:

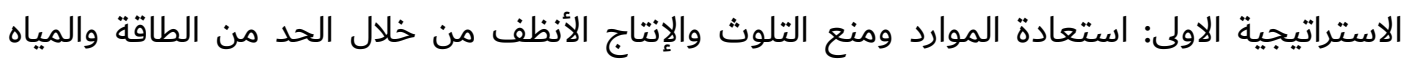

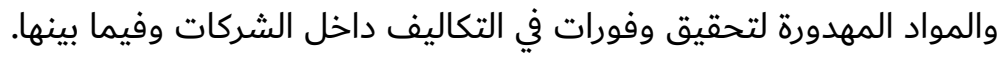

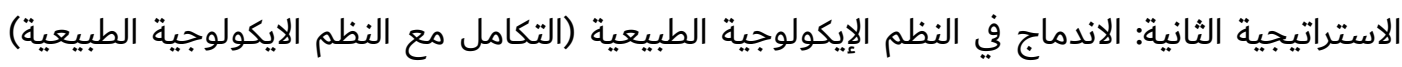

• تحديد القدرة الاستيعابية للموقع، والتصميم ضمن تلك الحدود. • الحفاظ على المناطق الطبيعية والنباتات الأصلية قدر الإمكان. • الاحتفاظ بنظم الصرف الطبيعية واستخدام الأراضي الرطبة المبنية أو الطبيعية لتنقية المياه الصناعية أو العواصف.

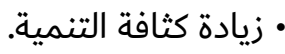
• • تصميم المواقع والمباني ذات الكفاءة في استخدام الطاقة. • توقيع الشركات لتحقيق خدمة أسهل والتعايش الصناعي. الاستراتيجية الثالثة: التجمعات الصناعية: عبارة عن شبكات من المصنعين تطور علاقات تعاونية لتحسين الموارد عن طريق التجميع على طول سلسلة قيمة كاملة. الاستراتيجية ع: تصميم مستدام (أخضر) وتتضمن: زيادة كفاءة الطاقة من خلال تصميم المرافق أو إعادة التهاد

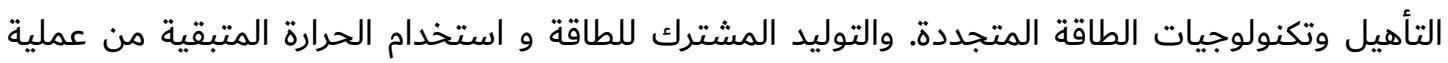
أولية لتوفير التدفئة أو التبريد لعملية لاحقة.لتصميم المباني المرنة للاستخدامات المات المتعددة.

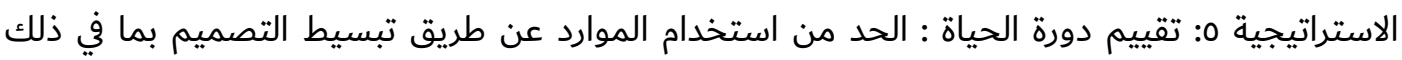

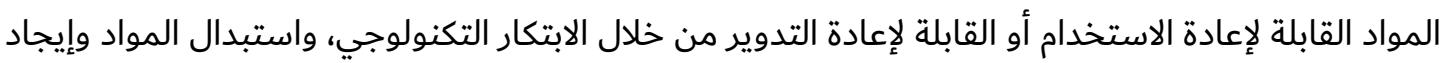

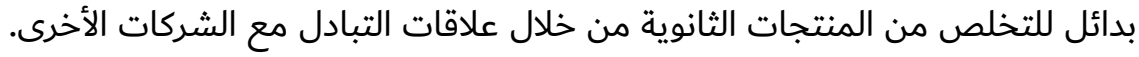
الاستراتيجية ا: التدريب المهني: حسين كفاءة استخدام اليد العاملة من خلال التأكيد على تطوير برامج التدريب على المهارات المشتركة للسكان المحليين.

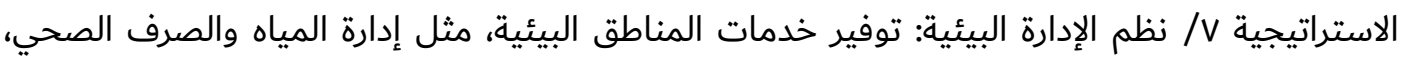

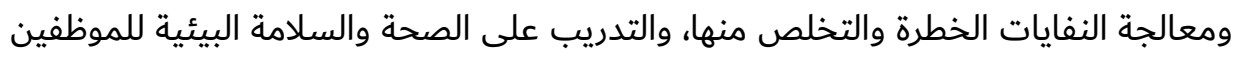

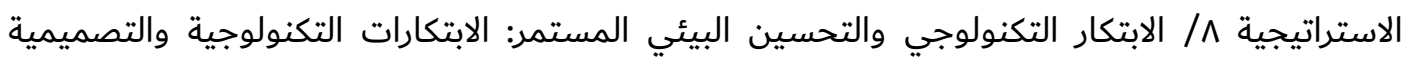

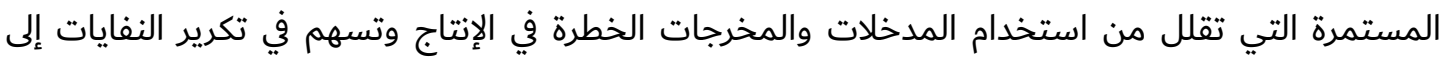
نوعية ملائمة لتصبح مدخلات. الاستراتيجية و/ المشاركة العامة والتعاون: اعتماد أدوات التخطيط المجتمعية لبناء العلاقات وإبلاغ جهود 


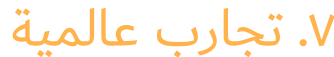

1-V

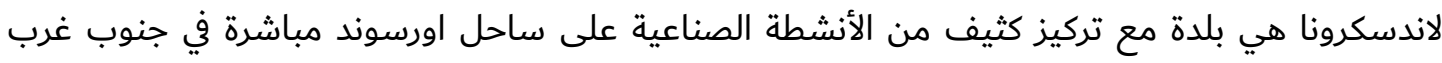

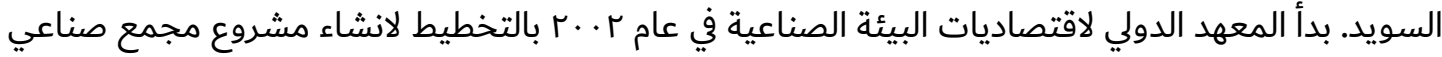

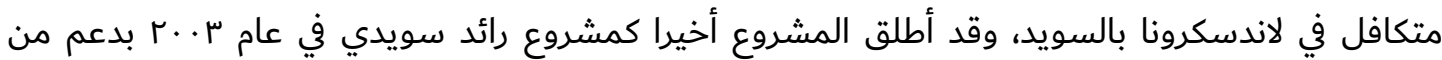

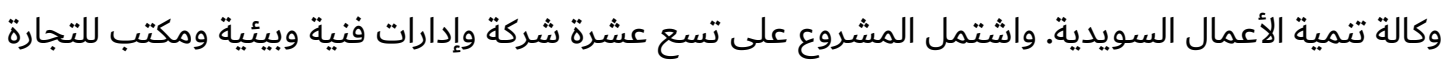

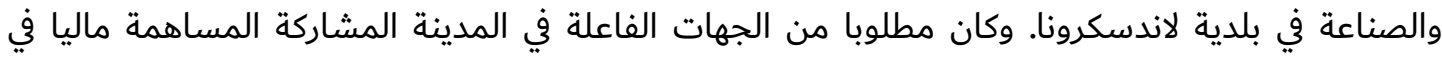

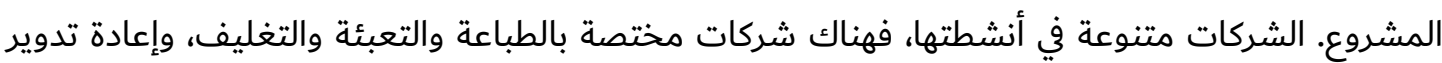

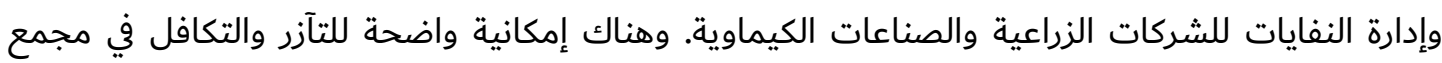

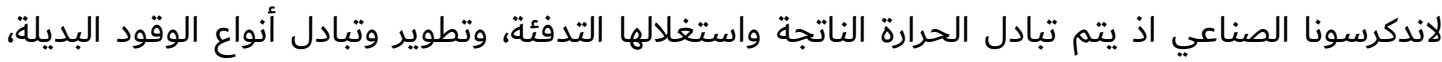

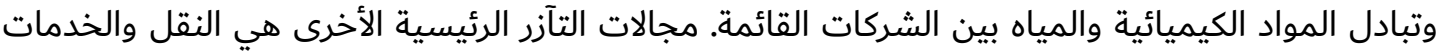

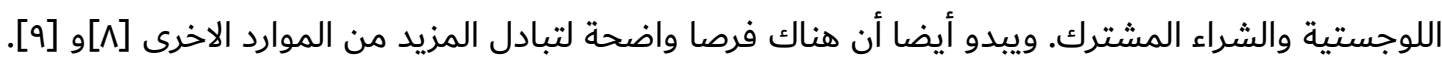

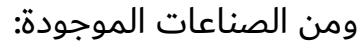

أكثر من عشرين شركة من مختلف القطاعات: المواد الكيميائية، وإدارة النفايات، ومعالجة المعادن وإعادة

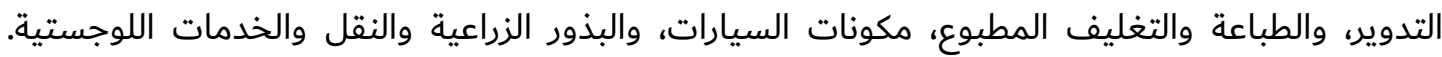
والمنظمات العامة المهتمة بإدارة البنية التحتية الرئيسية، والشؤون البيئية، وتنمية الأعمال التجارية.

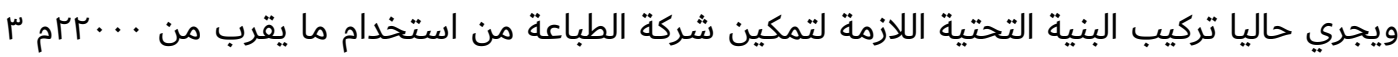
/ سنة من مياه الصرف الصحي الناتجة من مصنع لتصنيع زجاج السيارات المجاور في نظام تنظيف غازات

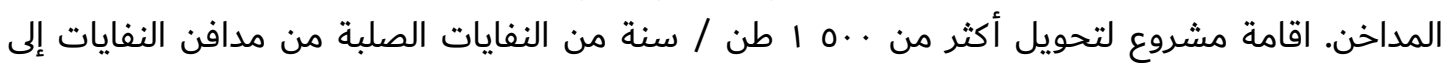

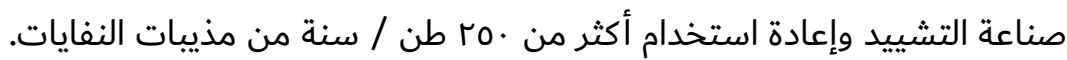

\section{V-V r-V خطة مدينة شيكاغو للصناعات المستدامة}

ان خطة عمل للتصنيع جاءت نتيجة تعاون مدته ثلاث سنوات من قبل قادة الصناعة والوكالات الحكومية

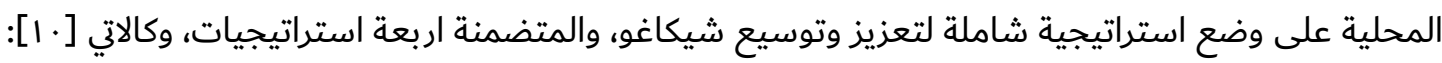

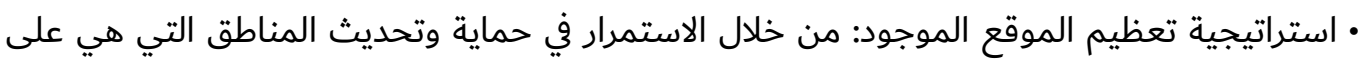
وجه التحديد بنيت للتصنيع والاستخدامات ذات الصلة. • استراتيجية الاستفادة من الخدمات اللوجستية المحلية: عن طريق رفع مستوى خدمات شحن، ميناء،

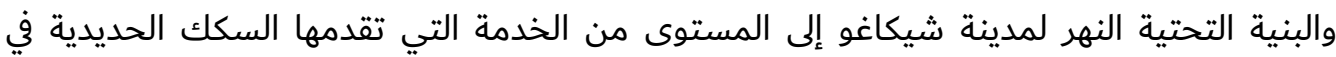
المدينة والهواء والبنية التحتية مع دمج دور المستودعات ومرافق التوزيع. • استراتيجية تعظيم البنية التحتية للمرافق : من خلال معالجة خدمات المتعلقة بالمنطقة الصناعية

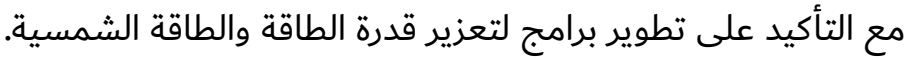
• استراتيجية إنشاء تعاونية التصنيع: بواسطة إشراك الحكومة المحلية، الشركات المصنعة، والقوى العاملة والمنظمات. 
United Nation of indus- (منظمة الامم المتحدة للتنمية الصناعية (Unido) (r-V المثتدام الصناعة الخضراء للتنمية الصناعية

تناولت هذه المبادرة لتحقيق الاستدامة في الصناعات القائمة والصناعات الجديدة، اذا ان الصناعة الخضراء

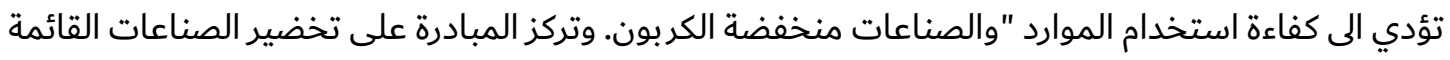

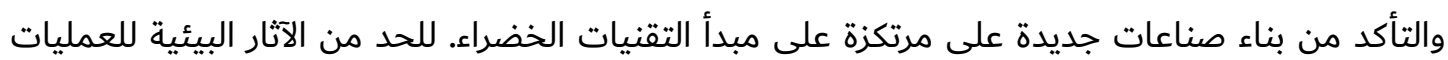
والمنتجات [11 [11]. كما تناولت العلاقة بين الصناعة الخضراء والاستدامة، اذ ان الصناعة الخضراء تكون على المستوى الكلي

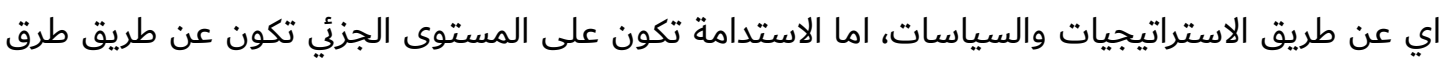

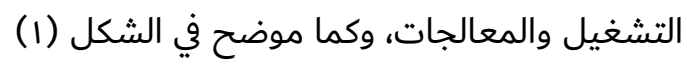

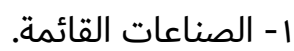
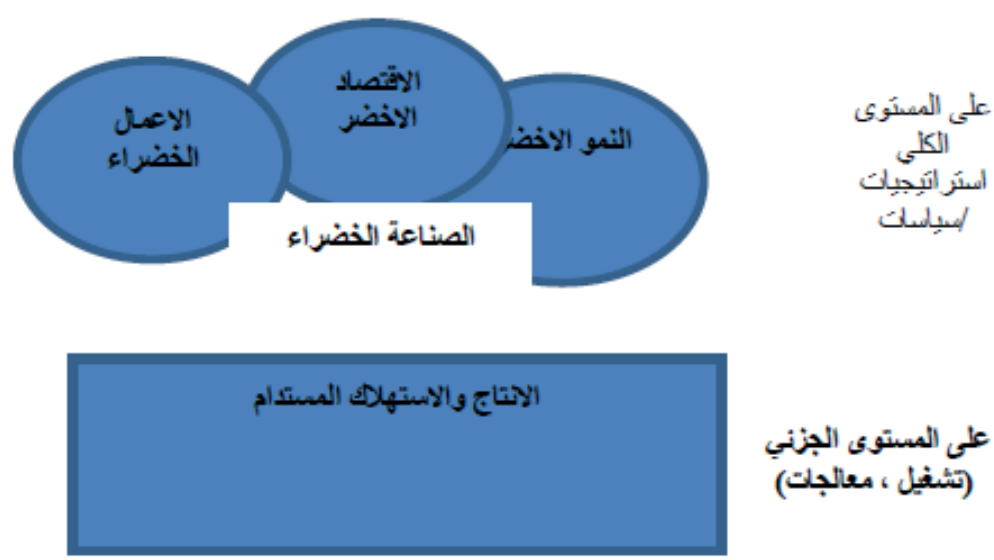
(تشفيل ، معالجنت)

شكل ا: الصناعة الخضراء هي استراتيجية قطاعية لتحقيق النمو الأخضر - الاقتصاد الأخضر - الوظائف الخضراء في الصناعات التحويلية والقطاعات لتحقية ذات الأخدات الصلة.

أ- الصناعة الخضراء في الصناعات القائمة يتم ذلك عن طريق تخضير الصناعة Greening of industry: باتباع طرق الوقاية من التلوث، ومتابعة

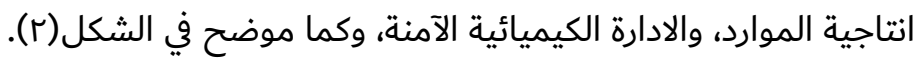
ب- الصناعات الخضراء في الصناعات الجديدة. يتم ذلك من خلال خلق الصناعات الخضراء من خلال الخدمات البيئية والتقنيات الخضراء، وكما موضح في الشكل (r). (r). يتم ذلك عن طريق تخضير الصناعة Greening of industry: باتباع طرق الوقاية من التلوث، ومتابعة

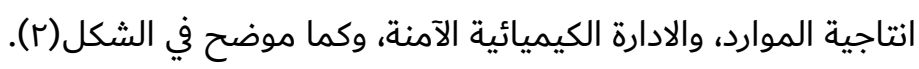
التأجير الكيميائي Chemical leasing: هو نموذج الأعمال الذي تقوم فيهايه التيكاتيه الشركة الكيميائية بتزويد

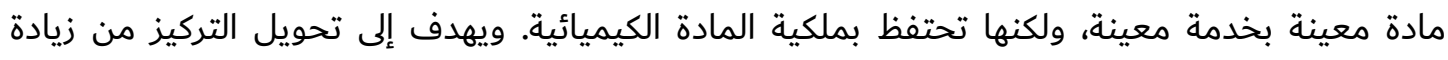

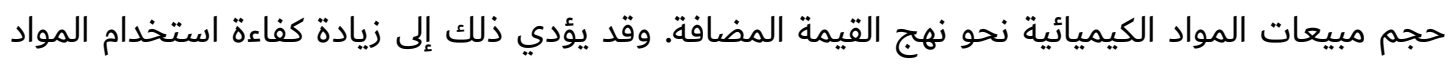
الكيميائية، وإلى المنافع النوعية والبيئية والاقتصادية. 


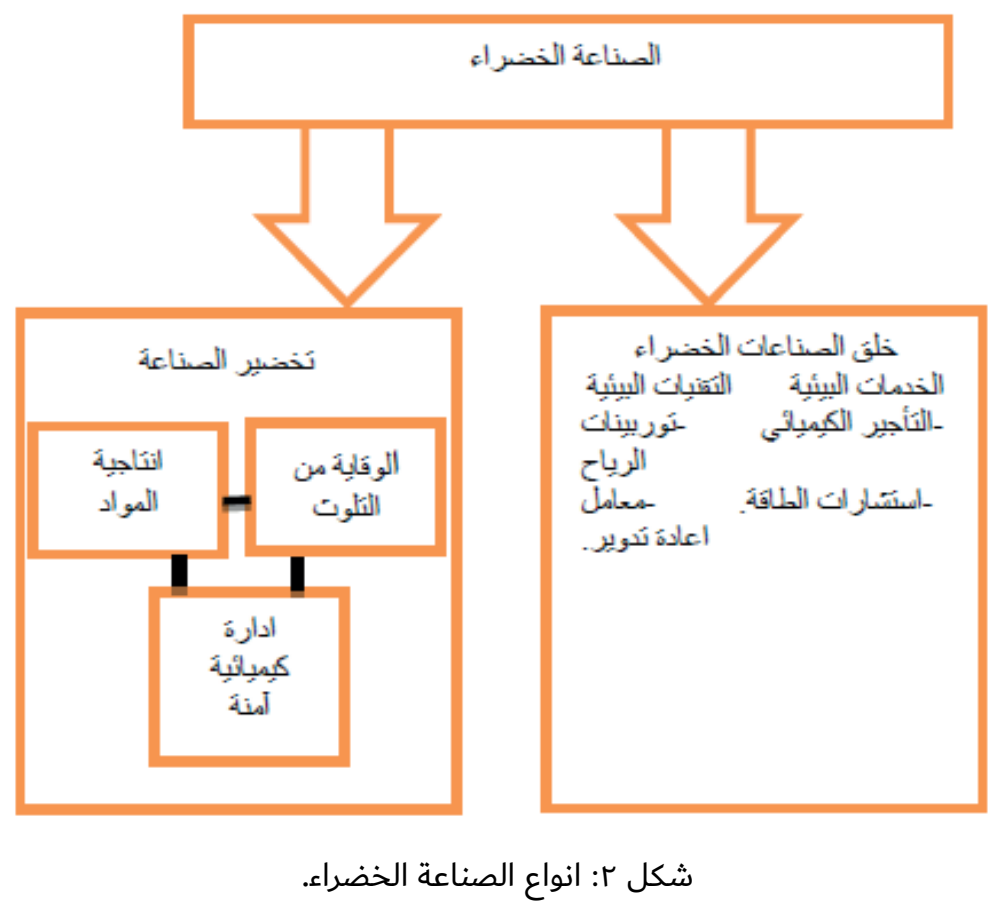

\section{^. التوجهات في العراق نحو انشاء المدن الصناعية المستدامة}

تضمنت الاستراتيجية الصناعية للعراق حتى عام •ـr.r العمل على بناء بناء نواة تجمعات صناعية تنافسية

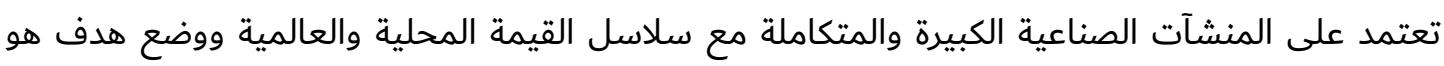

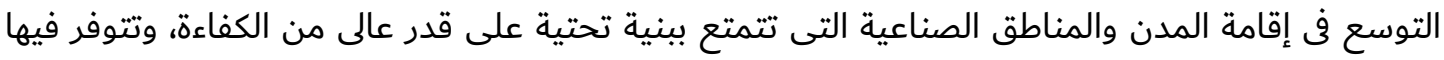
الحوافز للتصنيع وذلك من خلال خطة تنفذ على ثلاثة مراحل هي [r Ir]: المرحلة الاولى عام VIV·r: يتم بناء 0 مدن صناعية جديدة والاستمرار بتنفيذ مدينة خور الزبير الصناعية الكبرى للصناعات كثيفة الطاقة. المرحلة الثانية عام r ·r r. بناء V مدن صناعية إضافية ومدينة تكنولوجية واحدة.

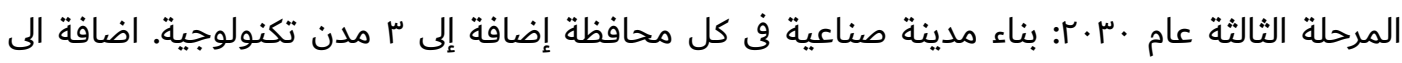

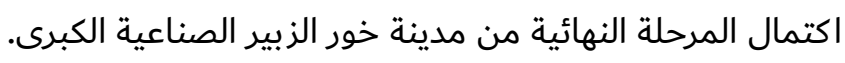

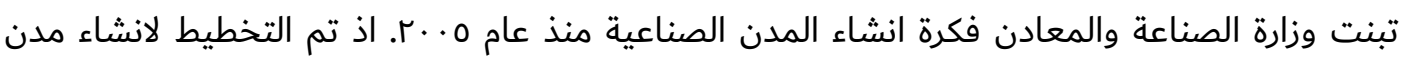

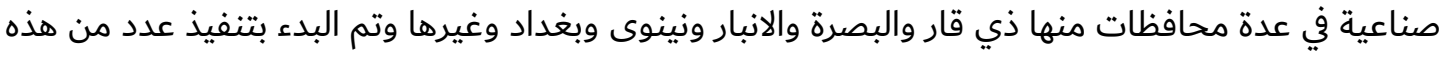
المدن وحسب الخريطة رقم (1) ادناه والتي توضح مواقع المدن المقترحة ومراحل تقدم العمل. ومن خلال تحليل المقترحات المتعلقة بهذه المدن الصناعية والاستراتيجيات المقترحة لتنفيذها نجد ان ان

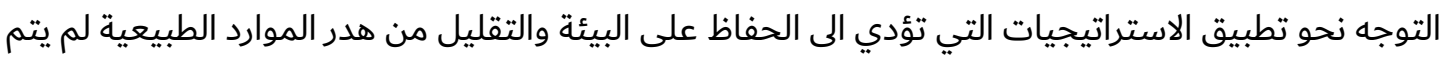

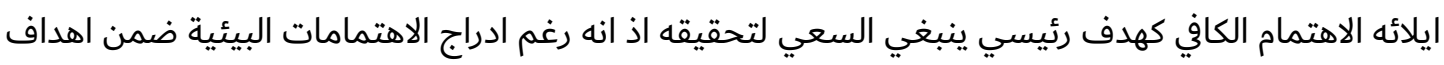

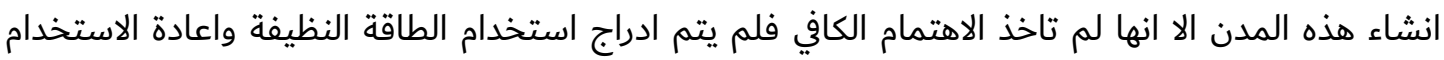

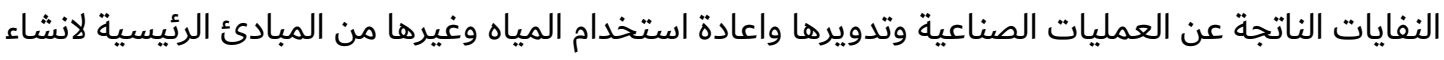

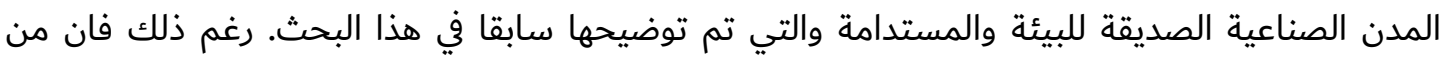




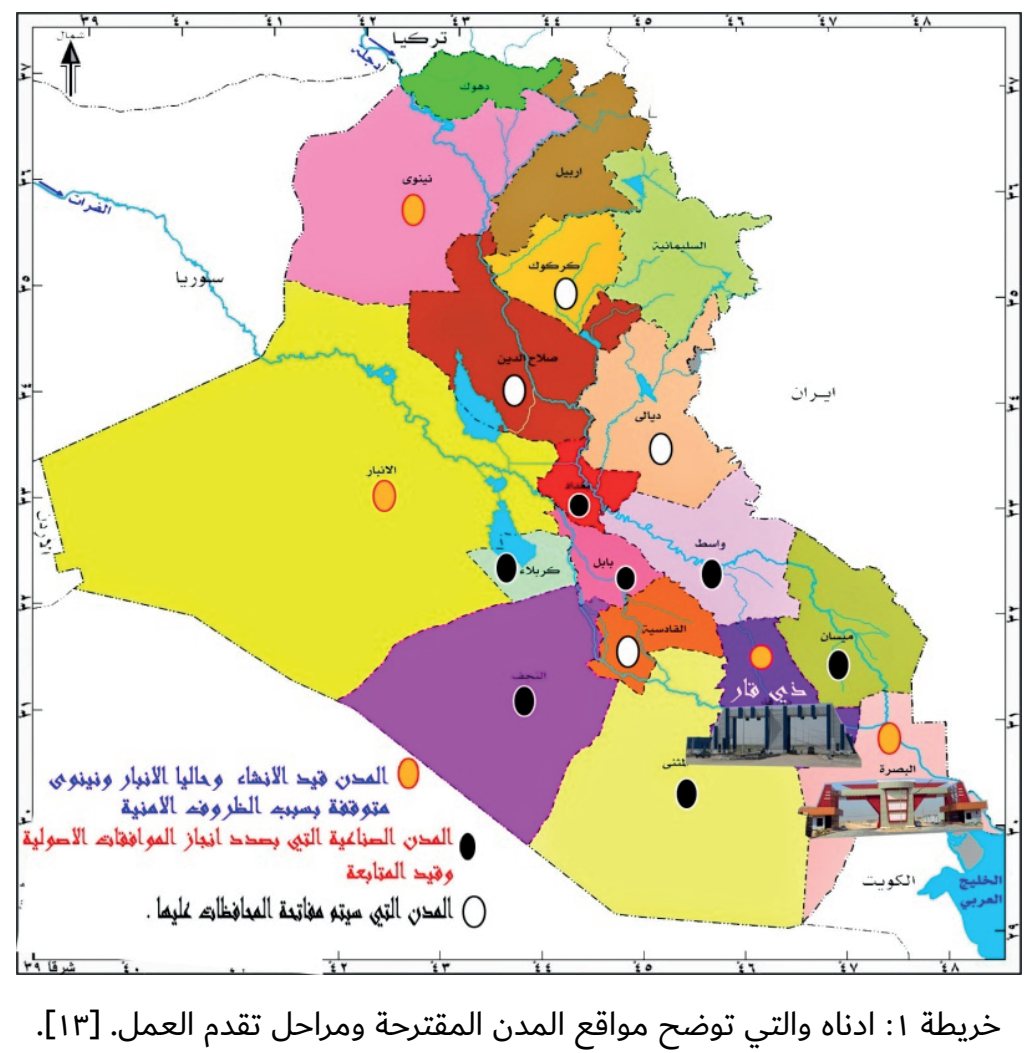

النقاط الجيدة هو التوجه نحو انشاء وحدات لمعالجة المياه الثقيلة والنفايات ومحطات للكهرباء وللتصفية لكن يجب ان يتم تحديد اليات عمل هذه المحطات ان يكون وفقا لمبادئ الاستدامة وكما تم توضيحه اعلاه.

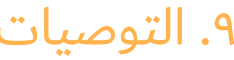

على توجيهات الصناعة في العراق فان التوجه نحو تفعيل المناطق الصناعية المستدامة يمكن من خلال اتباع ما يلي:

• في مجال الصناعة يمكن تفعيل مبادرة (UNIDO) فيما يخص الصناعات القائمة باتباع استراتيجيات خلق الصناعات الخضراء من خلال توفير الخدمات والتقنيات البيئية، اما فيما يخص الصناعات الجديدة فيمكن اتباع استراتيجيات تخضير الصناعة.

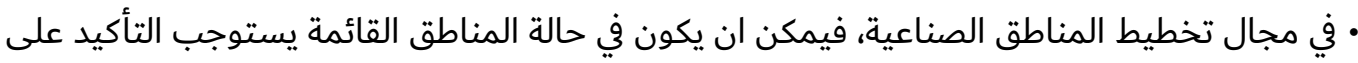

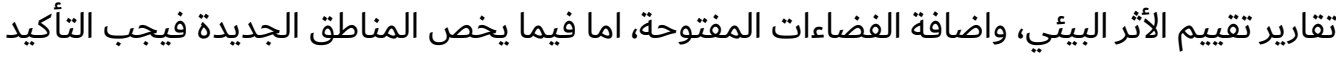
على كفاءة العلاقة بين المبنى الصناعي والاستعمالات المجاورة والمساحات.

[1] Organazation for economic co-operartion and development, " The application of biotechnology to industrial sustainablity-A primer". 
[2] Internationale Zusammenarbeit (GIZ), "Guidelines for Sustainable Industrial Areas (SIA)," v 1.0,), Eschborn, October 2015.

[3] C, R.P, Ellison, R, "Designing and Operating Industrial Parks as Ecosystems," Dalhousie University, School for Resource and Environmental Studies: Halifax, NS, Canada, 1994.

[4] E. Conticelli, S. Tondelli, "Application of Strategic Environmental Assessment to EcoIndustrial Parks: Raibano Case in Italy," J. Urban Plan. Dev. 2013, 139, 185-196.

[5] E. Lowe, J. Warren, "The Source of Value: An Executive Briefing and Sourcebook on Industrial Ecology; PNNL-10943 Pacific Northwest National Laboratory," Richland, DC, USA, 1996.

[6] 4-Elisa Conticelli *and Simona Tondelli," Eco-Industrial Parks and Sustainable Spatial Planning: A Possible Contradiction?," Administrative Sciences, vol. 4, issue 3, 2014.

[7] M. Schlarb, "Eco-industrial Development: A Strategy for Building Sustainable Communities," United States Economic Development Administration, Cornell University: Washington, DC, USA, 2001.

[8] M. Mirata, T. Emtairah, "Industrial symbiosis networks and the contribution to environmental innovation: The case of the Landskrona Industrial Symbiosis Programme," Journal of Cleaner Production 13,993-1002.

[9] M. Maltin, "Industrial symbiosis and its alignement with regional Sustainability, " exploring the possibilities in Landskrona, Sweden. Lund IIIEE Reports 200:7. Lund University.

J. Starlander, "Industrial Symbiosis: A Closer Look on Organisational Factors. A study based on the Industrial Symbiosis project in

Landskrona, Sweden. IIIEE Reports 2003:4. Masters Thesis. The Internationallnstitute for Industrial Environmental Economics. Environmental Management and Policy. Lund, Sweden.

[10] M. Rahm, "Chicago sustainable industies Abusiness plan for manufacturing city of Chicago," November 2013.

[11] UNIDO, "Green Industry Policies for supporting Green Industry, " Vienna, May 2011.

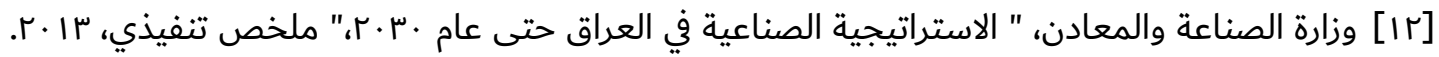

[13] http://www.gdid.gov.iq/index.php?name=Pages\&op=page\&pid=480

\section{References}

[1] Organazation for economic co-operartion and development, "The application of biotechnology to industrial sustainablity-A primer". 
[2] Internationale Zusammenarbeit (GIZ), "Guidelines for Sustainable Industrial Areas (SIA)," v 1.0, ), Eschborn, October 2015.

[3] C, R.P, Ellison, R, "Designing and Operating Industrial Parks as Ecosystems," Dalhousie University, School for Resource and Environmental Studies: Halifax, NS, Canada, 1994.

[4] E. Conticelli, S. Tondelli, "Application of Strategic Environmental Assessment to Eco-Industrial Parks: Raibano Case in Italy," J. Urban Plan. Dev. 2013, 139, 185-196.

[5] E. Lowe, J. Warren, "The Source of Value: An Executive Briefing and Sourcebook on Industrial Ecology; PNNL-10943 Pacific Northwest National Laboratory," Richland, DC, USA, $19 ; 96$.

[6] Elisa Conticelli *and Simona Tondelli, "Eco-Industrial Parks and Sustainable Spatial Planning: A Possible Contradiction?," Administrative Sciences, vol. 4, issue 3, 2014.

[7] M. Schlarb, "Eco-industrial Development: A Strategy for Building Sustainable Communities, "United States Economic Development Administration, Cornell University: Washington, DC, USA, 2001.

[8] M. Mirata, T. Emtairah, "Industrial symbiosis networks and the contribution to environmental innovation: The case of the Landskrona Industrial Symbiosis Programme," Journal of Cleaner Production 13,993-1002.

[9] M. Maltin, "Industrial symbiosis and its alignement with regional Sustainability," exploring the possibilities in Landskrona, Sweden. Lund IIIEE Reports 200:7. Lund University. J. Starlander, "Industrial Symbiosis: A Closer Look on Organisational Factors. A study based on the Industrial Symbiosis project in Landskrona, Sweden. IIIEE Reports 2003:4. Masters Thesis. The Internationallnstitute for Industrial Environmental Economics. Environmental Management and Policy. Lund, Sweden.

[10] M. Rahm, "Chicago sustainable industies Abusiness plan for manufacturing city of Chicago," November 2013.

[11] UNIDO, "Green Industry Policies for supporting Green Industry," Vienna, May 2011.

[12] Ministry of Industry and Minerals, "Industrial Strategy in Iraq to 2030," Executive Summary, 2013.

[13] http://www.gdid.gov.iq/index.php?name=Pages\&op=page\&pid=480. 